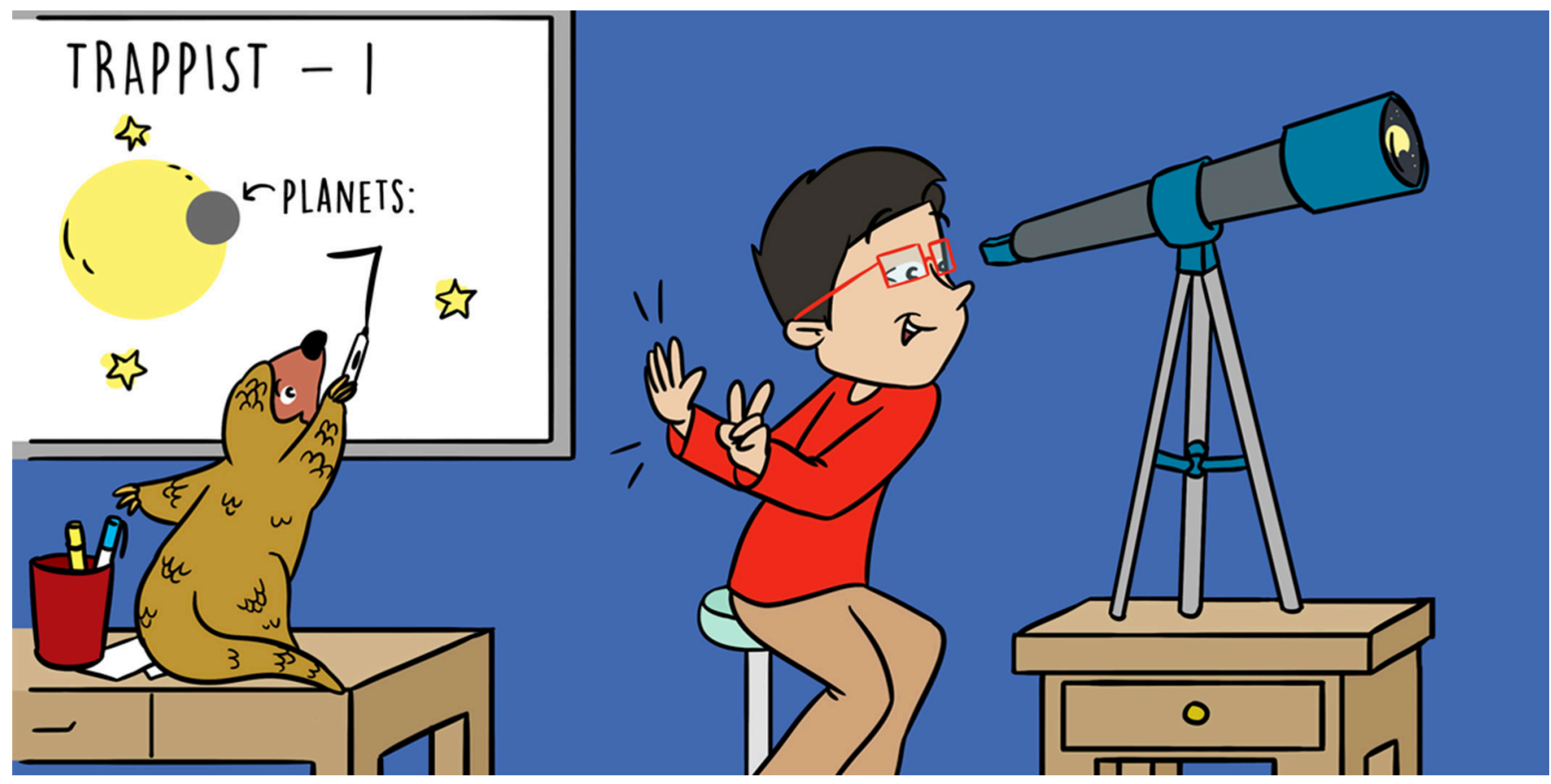

\title{
TRAPPIST=1: A DARK STAR WITH A BRIGHT FUTURE
}

\section{Brett M. Morris *}

Astronomy Department, University of Washington, Seattle, WA, United States

YOUNG REVIEWERS:

STEVENSON

PACT

ELEMENTARY

SCHOOL

AGE: $12-15$

\section{HOST STAR}

The star that an exoplanet orbits.

\section{EXOPLANET}

A planet orbiting a star other than the Sun.
Of the thousands of stars known to host exoplanets, which are planets outside of our solar system, a particularly fascinating star stands out. It is known as TRAPPIST-1: a tiny star about the size of Jupiter, which is home to not one, not two, but seven Earth-sized planets! These planets are probably rocky worlds, like earth, and some of them might have the correct surface temperature for liquid water to exist, but that depends on whether or not these planets have atmospheres, and what those atmospheres are made of. Astronomers are currently working on figuring out whether TRAPPIST-1 has bright or dark spots on it, which may affect the way we see its planets.

\section{DISCOVERING FAR-AWAY PLANETS}

Most stars have planets orbiting them. These stars are called host stars, and exoplanets are what we call planets that orbit other stars, outside of our own solar system. If we view an exoplanet system from the correct angle, the exoplanets will appear to eclipse their host star as they move around the star on their orbits. If we measure the brightness of the star with excellent precision when it is eclipsing its star, we 


\section{TRANSIT}

When an exoplanet blocks out light from its host star.

\section{LIGHT YEAR}

The distance light travels in 1 year.

\section{FUSION}

The nuclear reaction that occurs in the cores of stars, which makes them shine.

\section{LUMINOSITY}

The total brightness of an astronomical object. can observe a transit event, which is described in detail in this Frontiers for Young Minds article. During a transit, a planet blocks out some starlight, and astronomers on Earth see a dip in the total amount of starlight, which typically lasts for a few hours (Figure 1).

TRAPPIST-1 is a very small, red star located 40 light years away, in the constellation Aquarius. In fact, TRAPPIST-1 is about as small as a star could be-if it were a bit smaller, it would not even be considered a star, because it would not be big enough to produce its own light by fusing hydrogen into helium in its core. TRAPPIST-1 is about as big as the planet Jupiter, but it weighs 80 times as much as Jupiter. It shines with only $0.05 \%$ the luminosity of the Sun [1]. Most of stars in the galaxy are small stars like TRAPPIST-1, and since they are so small and so dim, they are challenging to study.

\section{SEVEN SMALL PLANETS}

These challenges did not stop one team of astronomers based in Belgium from trying to find planets orbiting TRAPPIST-1, and what a zoo of planets they found! There are seven known exoplanets orbiting this tiny star, each of them passing in front of the star when viewed from Earth, causing transit events. We can figure out how large each planet is by measuring how much light goes missing when the planet passes in front of the star. Small planets block out a little bit of light, while bigger planets block out more light. The brightness measurements of TRAPPIST-1 indicated that these planets have sizes similar to Earth-the smallest planet is three-quarters the size of Earth, and the largest is about 13\% larger than Earth (see Figure 2) [1].

The TRAPPIST-1 planets orbit their host star much more closely than planets in our solar system orbit the Sun. The closest planet, called TRAPPIST-1 b, completes one orbit around its star once every 1.5 days. Compare that with Earth, which takes a year to orbit the Sun once. The most distant planet, TRAPPIST-1 h, completes an orbit every 18 days. If you were to put all of the TRAPPIST-1 planets into our solar system, their orbits would all fit within the orbit of Mercury, the solar system's innermost planet.

\section{COULD LIQUID WATER (AND PERHAPS LIFE) EXIST ON ANY OF THE PLANETS?}

You might be wondering whether these planets are too close to the star for humans to visit them without melting. It turns out that, since the star TRAPPIST-1 is so dim, the planets need to be very close to the star to keep them warm. Stars work just like campfires-the closer a planet is to a star, the warmer it will probably be. But the distance from a star is not the only factor that determines how warm a planet is. 
Figure 1

\section{An artist's}

interpretation of what the TRAPPIST-1 system might look like. Seven planets orbit this star. The dark circles on the star are the shadows of planets passing in front of the star. Image credit:

NASA/JPL-Caltech.
Figure 2

An artist's interpretation of the TRAPPIST-1 planets, called $b-h$, and their host star, with their sizes shown to scale (though the distances are not to scale). Image credit: NASA/JPL-Caltech.

\section{ATMOSPHERE}

\section{A layer of gas} surrounding a planet (like Earth's air).

\section{GREENHOUSE}

\section{EFFECT}

When a planet's atmosphere is made of a gas that traps heat, increasing the planet's surface temperature.
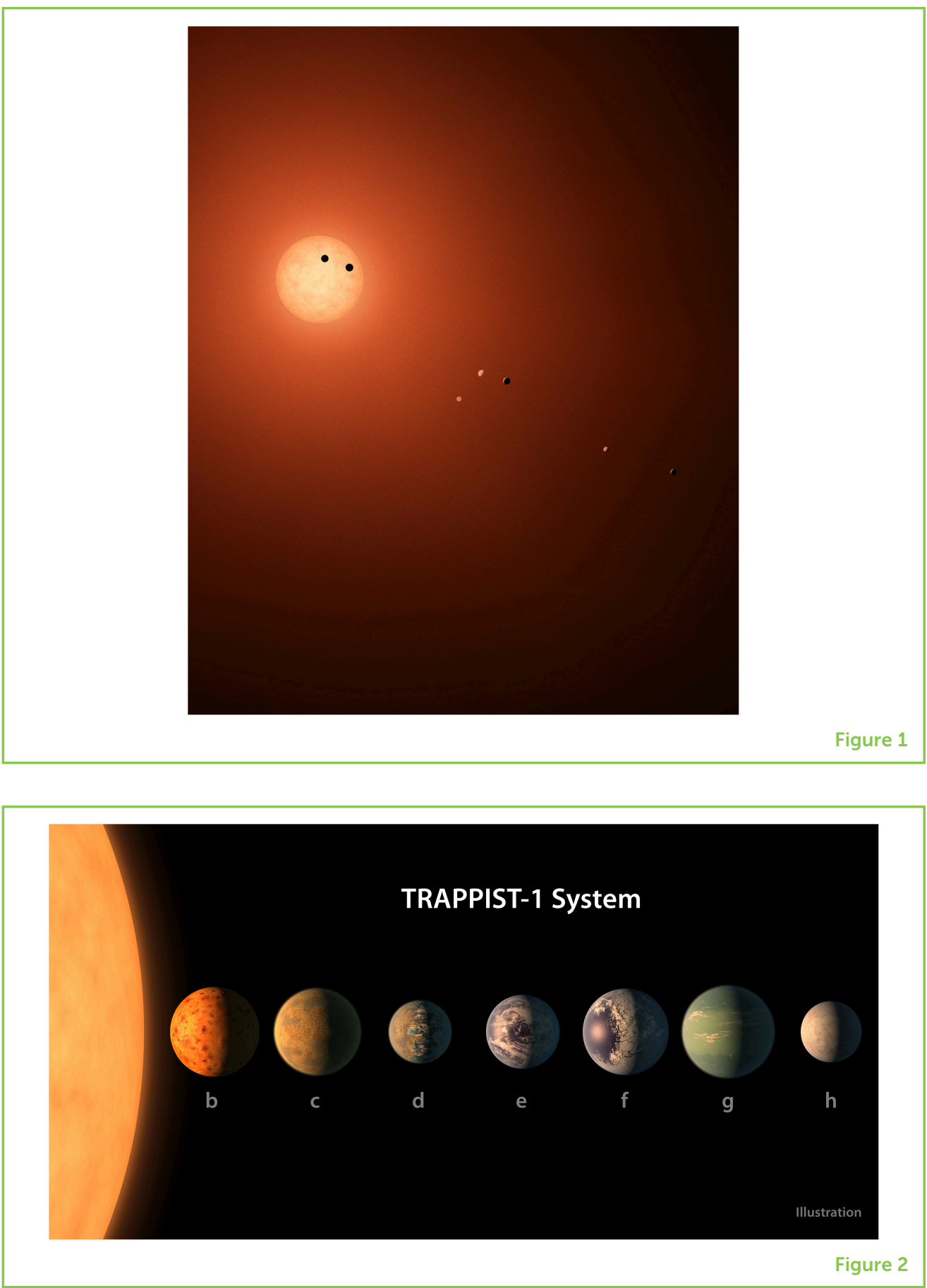

The atmosphere of the planet is also important to consider, because some atmospheres keep their planets extra warm and toasty. Mercury and Venus are good examples of the warming effect of atmospheres. Mercury is two times closer to the Sun than Venus, so you might guess that Mercury would be the hottest planet in our solar system. But Mercury's highest surface temperature is about $430^{\circ} \mathrm{C}\left(800^{\circ} \mathrm{F}\right)$, while Venus' surface temperature is even hotter, at about $460^{\circ} \mathrm{C}\left(860^{\circ} \mathrm{F}\right)$. The reason Venus is hotter than Mercury is because its atmosphere is made mostly of carbon dioxide, which traps heat in Venus' atmosphere, in a phenomenon called the greenhouse effect. Mercury does not 
have an atmosphere, so there is no greenhouse effect there, and the temperature does not rise as high as it does on Venus.

The surface temperature of each of the TRAPPIST-1 planets will depend on whether or not they have atmospheres, and what those atmospheres are made of. Right now, we do not know for sure if any of the TRAPPIST-1 planets have atmospheres, and there is a wide variety of possible atmospheres that could exist on each of the planets, each of which could have a significant impact on how warm each planet might be.

As a result of these questions about the atmospheres, we do not yet know the surface temperatures for each of the TRAPPIST-1 planets. We also do not know for sure whether or not any of them could have liquid water on their surfaces. But we intend to find out!

One way we can find out is by watching each planet pass in front of the star-planets with atmospheres block out more light of certain colors than planets without atmospheres, and which colors are blocked depends on the chemicals present in the planet's atmosphere. By measuring the color of the star very carefully when the planet passes in front of the star, we may be able to determine if the planets have atmospheres, and what those atmospheres are made of.

\section{THE MYSTERIOUS SPOTS ON THE SURFACE OF TRAPPIST-1}

However, there is a major challenge making it difficult to study the atmospheres of the TRAPPIST-1 planets-we do not know what the surface of the star looks like. The star could have large or small spots on its surface, called starspots, which affect the brightness and color measurements that we take of the star [2]. Since we rely on those brightness and color measurements to learn about the planets' atmospheres, and therefore whether or not the planets could have liquid water, we must know precisely how spots on TRAPPIST-1 affect the color and brightness of that star.

One effort to study the starspots of TRAPPIST-1 using NASA's Kepler and Spitzer space telescopes found that the star might not have dark starspots like the Sun at all, but it might have bright, hot spots on its surface [3]. These bright spots make the star appear a bit bluer and brighter when the bright spots are facing us, and a bit redder and dimmer when the bright spots rotate out of view as the star spins. Figuring out the number, temperature and brightness of these spots on the star will be important for understanding the imprints that the planets leave on the starlight from TRAPPIST-1. Recently, two other groups of scientists studying the color of TRAPPIST-1 also concluded that there may be bright (hot) spots on its surface [4, 5]. As the evidence builds for these strange spots, so does the mystery surrounding them, 
since the Sun-the only star we can study up close-does not have similarly bright hot spots on its surface.

\section{A NEW EYE ON THE SKY}

Astronomers will likely get answers to their questions about the spots of TRAPPIST-1 when a new space telescope launches, called the James Webb Space Telescope (JWST). The JWST will be the largest space telescope ever built, and it is scheduled to observe the TRAPPIST-1 planets early in its 5 -years mission. In combination with observations taken by telescopes on the Earth, astronomers will use the observations from the JWST to finally figure out what the surface of TRAPPIST-1 looks like, and then, hopefully, whether or not the TRAPPIST-1 planets have atmospheres and could be hospitable to life as we know it.

\section{REFERENCES}

1. Gillon, M., Triaud, A. H. M. J., Demory, B.-O., Jehin, E., Agol, E., Deck, K. M., et al. 2017. Seven temperate terrestrial planets around the nearby ultracool dwarf star TRAPPIST-1. Nature. 542:456-60. doi: 10.1038/nature21360

2. Rackham, B. V., Apai, D., and Giampapa, M. S. 2018. The transit light source effect: false spectral features and incorrect densities for $M$-dwarf transiting planets. Astrophys. J. 853:122. doi: 10.3847/1538-4357/aaa08c

3. Morris, B. M., Agol, E., Davenport, J. R. A., and Hawley, S. L. 2018. Possible bright starspots on TRAPPIST-1. Astrophys. J. 857:39. doi: 10.3847/1538-4357/aab6a5

4. Ducrot, E., Sestovic, M., Morris, B. M., Gillon, M., Triaud, A. H. M. J., De Wit, J., et al. 2018. The $0.8-4.5 \mu \mathrm{m}$ broadband transmission spectra of TRAPPIST-1 planets. Astron. J. 156:218. doi: 10.3847/1538-3881/aade94

5. Wakeford, H. R., Lewis, N. K., Fowler, J., Bruno, G., Wilson, T. J., Moran, S. E., et al. 2019. Disentangling the planet from the star in late-type $M$ dwarfs: a case study of TRAPPIST-1g. Astron. J. 157:11. doi: 10.3847/1538-3881/aaf04d

SUBMITTED: 21 January 2019; ACCEPTED: 25 March 2019;

PUBLISHED ONLINE: 10 April 2019.

EDITED BY: Edward Gomez, Las Cumbres Observatory Global Telescope Network, United States

CITATION: Morris BM (2019) TRAPPIST-1: A Dark Star With a Bright Future. Front. Young Minds 7:56. doi: 10.3389/frym.2019.00056

CONFLICT OF INTEREST STATEMENT: The author declares that the research was conducted in the absence of any commercial or financial relationships that could be construed as a potential conflict of interest.

COPYRIGHT (c) 2019 Morris. This is an open-access article distributed under the terms of the Creative Commons Attribution License (CC BY). The use, distribution 


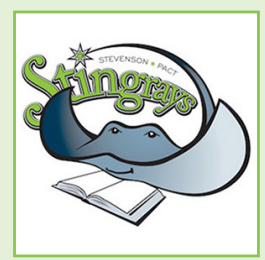

or reproduction in other forums is permitted, provided the original author(s) and the copyright owner(s) are credited and that the original publication in this journal is cited, in accordance with accepted academic practice. No use, distribution or reproduction is permitted which does not comply with these terms.

\section{YOUNG REVIEWERS}

STEVENSON PACT ELEMENTARY SCHOOL, AGE: 12-15

We are the creative, fifth-grade, inclusive, fun, innovative, mature, responsible, energetic, involved in hands-on science, kind, generous, resilient, community-based, collaborative, enthusiastic students of Stevenson PACT! As writers, we work on finding facts and being specific with our note-taking strategies to improve our claims and scientific research.

\section{AUTHOR}

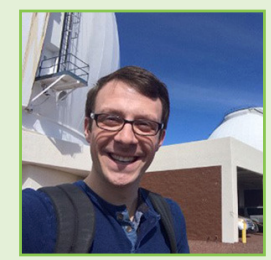

\section{BRETT M. MORRIS}

Brett Morris is a Ph.D. candidate at the University of Washington studying astronomy and astrobiology. Before that, Brett studied astronomy and physics at the University of Maryland and grew up on Long Island in New York. Brett has always wanted to be an astronomer, and is almost there! *morrisbrettmagmail.com 